\title{
The Influence of Adsorption Small Molecules Atrazine on Nonvolatile Resistive Switching Behavior in Co-Al Layered Double Hydroxide Films
}

Yanmei Sun ( 2017066@hlju.edu.cn )

Heilongjiang University https://orcid.org/0000-0002-9326-4560

Li Li

Heilongjiang University

Keying Shi

Heilongjiang University

\section{Nano Express}

Keywords: CoAl-LDHs, atrazine, resistive switching, memory device

Posted Date: September 18th, 2020

DOI: https://doi.org/10.21203/rs.3.rs-78844/v1

License: (1) This work is licensed under a Creative Commons Attribution 4.0 International License.

Read Full License

Version of Record: A version of this preprint was published at Journal of Materials Science: Materials in Electronics on March 9th, 2021. See the published version at https://doi.org/10.1007/s10854-021-05365-

Z. 


\title{
The Influence of Adsorption Small Molecules Atrazine on Nonvolatile Resistive
}

\author{
Switching Behavior in Co-Al Layered Double Hydroxide Films \\ Yanmei Sun ${ }^{1,2 *}$, Li Li ${ }^{1,3^{*}}$ \& Keying $\mathrm{Shi}^{1}$
}

\author{
${ }^{1}$ Key Laboratory of Functional Inorganic Material Chemistry, Ministry of Education, School of Chemistry and \\ Material Science, Heilongjiang University, Harbin 150080, PR China \\ ${ }^{2}$ School of Electronic Engineering, Heilongjiang University, Harbin, 150080, PR China. \\ ${ }^{3}$ Key Laboratory of Chemical Engineering Process \& Technology for High-efficiency Conversion, School of \\ Chemistry and Material Science, Heilongjiang University, Harbin 150080, PR China
}

ABSTRACT : Co-Al layered double hydroxides (LDHs) thin films were prepared by drop-casting process on ITO coated glass substrates. And then the small molecule atrazine was adsorbed on the Co-Al LDHs film by impregnation method. Current-voltage characteristics revealed nonvolatile resistive switching in Co-Al LDHs adsorbed atrazine films. The Influence of adsorption small molecules atrazine on nonvolatile resistive switching behavior in Co-Al LDHs Film has been investigated. By varying the atrazine adsorbed content in Co-Al LDHs thin films, the nonvolatile resistive switching behavior of device could be adjusted in a controlled way. Entirely different nonvolatile resistive switching characteristic, such as write-once read-many-times memory effect and rewritable memory effect are discriminable by the current-voltage curves.

KEYWORDS: CoAl-LDHs, atrazine, resistive switching, memory device

\section{Introduction}

Nonvolatile memory is the basic unit in a computer where data is stored to perform logical operations [1-4]. With the development of modern electronic technology and further miniaturization of silicon semiconductor devices, new memory technologies have been vigorously developed [5-8]. Among these, resistive random access memory (RRAM) is considered the best candidate to replace traditional flash memory technology [9-15].

Two-dimensional (2D) nanocomposite has realized more than one breakthroughs in material 
and device issues due to their unique structure stability and functionality [16-23]. In the last few years, the 2D transition metal dichalcogenides-based resistive switching memory devices have been studied [24-26]. Nevertheless, this material has no high self-selectivity, which prevents large-scale integration without use of additional transistors. The reliability of 2D transition metal dichalcogenides-based memory devices still poses a problem; moreover, the performance of device varies from laboratory to laboratory [27]. While pursuing the robust device performance of 2D materials, we also focus on the robust physical research of 2D materials.

Layered double hydroxides (LDHs), also well-known as hydrotalcite-like compounds, are inorganic layered materials which have aroused great concern because of their potential applications in adsorption, sensors, electrochemistry and bionanotechnology. The tunable metal ions in a large area without altering material structure and anion exchange properties of LDHs materials render it promising candidate as ion exchanger and absorbent [28]. In recent years, LDHs are increasingly used as biomedical materials. LDHs has a layered structure, a positively charged lamellae structure, exchangeable anions between the layers, and adjustable interlayer space. It not only has the characteristics of biological drug loading, but also has a good electronic channel building function. In this work, Co-Al LDHs is selected as the active layer and the high-speed electron transfer channel formed by its layered structure is used to realize the resistive switching effect. Furthermore, 2-chloro-4-ethylamino-6-isopropylamino-1,3,5-triazine (atrazine) is known as a n-type semiconductor, which belongs to the group of symmetric triazine derivatives, Due to the introduction of electron-withdrawing group level halogen atoms, they have good charge transfer ability, Moreover, the attraction of the positively charged main plate of $\mathrm{CO}-\mathrm{Al} \mathrm{LDHs}$ to the negatively charged chlorine ions of atrazine, makes it easy to adsorb atrazine on the surface of the CO-Al LDHs.

Make allowances for a large number of researches about resistive switching and memory effects [29-32], the influence of adsorbed level on the resistive switching behavior, which seems that further research is needed. It is reasonable to expect that adsorbed level will have a noticeable impact on 
charge transport processes, and consequently will have significant influence on device property [33].

In this work, the nonvolatile resistive switching behavior in Co-Al layered double hydroxide adsorbed atrazine films was reported. The nonvolatile resistive switching behaviors of the Co-Al LDHs adsorbed atrazine films could be adjusted by changing the adsorbed atrazine content in active films. write-once-read-many-times (WORM) memory and rewritable memory can be realized by adjusting the adsorbed atrazine content on Co-Al LDHs film.

\section{Experimental details}

Co-Al LDHs were synthesized as our previous work [34,35], atrazine (molecular weight: 217.70) was provided by Shanghai Fusheng Industrial Co., LTD. ITO glass with a size of $1 \mathrm{~cm} \times 2 \mathrm{~cm}$ was cleaned by ultrasonic in acetone, methanol and deionized water for 35 minutes successively, and then dried in the vacuum drying phase for standby use. The Co-Al LDHs active layers were prepared by drop-casting technology on ITO glass substrate and dried at $50{ }^{\circ} \mathrm{C}$ for $8 \mathrm{~h}$. The dry film was impregnated in $10 \mathrm{mg} / \mathrm{ml}$ atrazine aqueous solution, and then dried at $50{ }^{\circ} \mathrm{C}$ for overnight to form the Co-Al LDHs adsorbed atrazine active layer. Whereafter, the electrode Al matrix was deposited by vacuum evaporation with the help of the mask (100 $\mu \mathrm{m}$ in width, $200 \mu \mathrm{m}$ in length). The electrical properties of memory devices were characterized by Keithley 4200 Semiconductor parameter testing system. During measurement of current-voltage characteristics of the device, the bottom electrode was always grounded.

\section{Result and discussion}

Fig.1(a) depicts the chemical structure diagram of Co-Al LDHs. Co-Al LDHs have a typical brucite regular octahedron structure. The synusias are composed of metal-oxygen octahedron. Because the Co in the center of the octahedron is replaced by Al homocrystals with similar radius, there is a large amount of permanent positive charge. The electrical equilibrium is maintained between the layers by exchangeable anions. Fig.1(b) depicts the chemical structure of atrazine. Co-Al LDHs adsorbed atrazine were used as the resistive switching layer in the devices. Schematic 
device structure of the Co-Al LDHs adsorbed atrazine-based device was shown in Fig.1(c). The cross section of resistive switching layer before deposition of top electrode Al was characterized by scanning electron microscopy as shown in Fig. 1(d), it can be seen the thickness of resistive switching layer about $150 \mathrm{~nm}$.
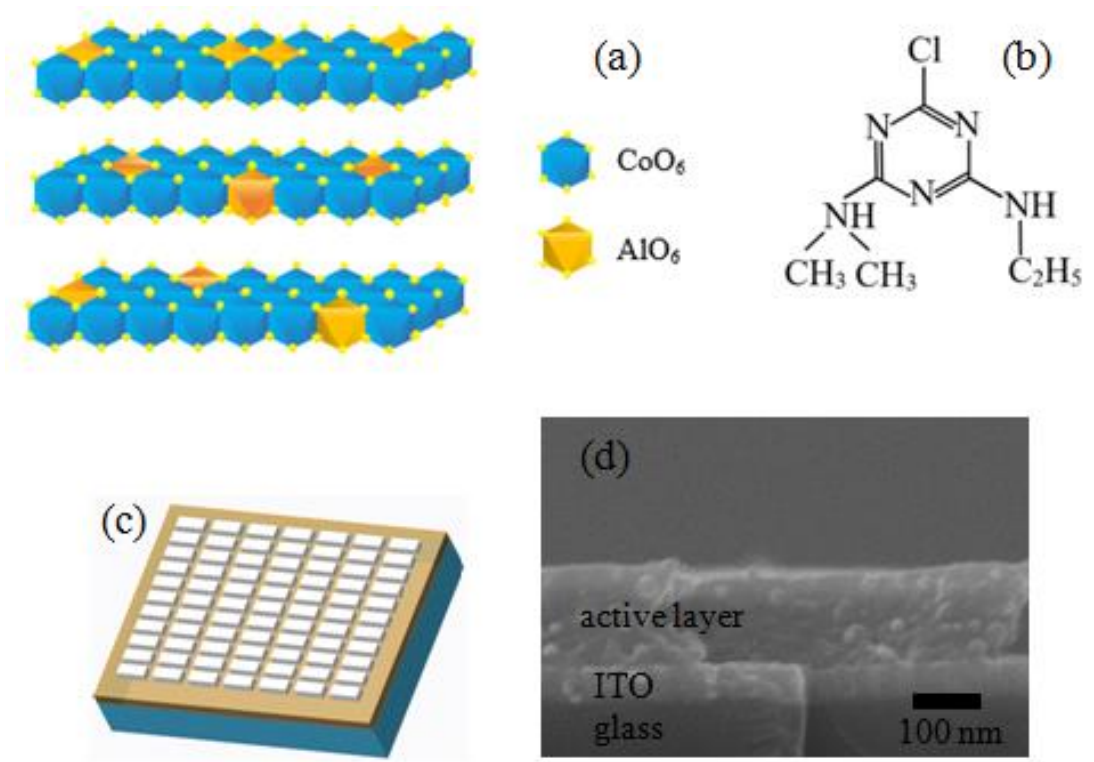

Fig. 1. (a) Chemical structure diagram of Co-Al LDHs. (b) Chemical structure of atrazine. (c) Schematic structure of the Co-Al LDHs adsorbed atrazine-based memory device. (d) The cross section of the resistive switching layer.

To study the influence of atrazine adsorption on the resistive switching characteristics of Co-Al LDHs film, the resistive switching characteristics of films with different impregnation times were studied. The immersion time of Co-Al LDHs film in atrazine aqueous solution was set as 3 min, 10 $\min , 15 \mathrm{~min}, 25 \mathrm{~min}, 30 \mathrm{~min}$ and $60 \mathrm{~min}$, respectively. The experimental results show that the resistive switching characteristics change significantly with the increasing of the impregnation time. The difference of the resistive switching depends on whether the impregnation time is greater or less than $15 \mathrm{~min}$. Therefore, the two devices are labeled Co-Al LDHs+Atrazine1(impregnation time is less than or equal to $15 \mathrm{~min}$ ) and Co-Al LDHs+Atrazine2 (impregnation time is greater than $15 \mathrm{~min}$ ) respectively.

To study the adsorbed atrazine influence on electrical properties of Co-Al LDHs-based memory 
device, the $I-V$ curves of the device was tested. Fig. 2(a) shows that the Co-Al LDHs+Atrazine1 memory device is initially at a high resistance state (HRS). The dc voltage scanning sequence applied to the device goes from $0 \mathrm{~V}$ to $+6 \mathrm{~V}$, from $0 \mathrm{~V}$ to $+6 \mathrm{~V}$, from $0 \mathrm{~V}$ to $-6 \mathrm{~V}$, and from $0 \mathrm{~V}$ to $-6 \mathrm{~V}$, the voltage sweep step is $0.01 \mathrm{~V}$. During the first positive voltage sweep from 0 to $+6 \mathrm{~V}$ (the first sweep), a sudden current increase took place at $1.22 \mathrm{~V}$, indicating the conversion of the device from the HRS to low resistance state (LRS), which amounts to a "writing" process in digital information storage, the switching threshold voltage of $V_{\text {set }}$ is $1.22 \mathrm{~V}$. During the succedent sweep2 from 0 to +6 $\mathrm{V}$, this device still remains in LRS and does not relax to HRS even turn off the power. Whereas, when the negative voltage scans from 0 to $-6 \mathrm{~V}$ (sweep3), a sudden current decrease took place at threshold voltage of $-4.81 \mathrm{~V}$, manifesting the conversion of the device from LRS to HRS, which equals to the "erasing" process in digital information storage. The same goes, the device holds on HRS during the succedent sweep4 and maintains in the HRS even after turn off the power. In addition, when the switching threshold voltage is reapplied, the device can be rewritten and reerased, manifesting that the device has a storage function, and could be reprogrammable. Fig. 2(b) depicts the cycling endurance of resistive switching characteristics under 176 consecutive DC voltage sweeps. The rewriting ability manifests that Co-Al LDHs+Atrazine1 memory device exhibits nonvolatile flash memory characteristics [36].

The cumulative probability for resistance in LRS and HRS were also collected for Co-Al LDHs+Atrazine1 device as depicted in Fig. 2(c). And the cumulative probability for threshold voltage of $V_{\text {set }}$ and $V_{\text {reset }}$ were also analyzed for Co-Al LDHs+Atrazine1 memory device as shown in Fig. 2(d), the average voltage required could be use to accomplish set and reset operations was stable.

Except for the I-V characteristics, other features for instance data retention characteristics and endurance characteristics under pulsed excitation are also critical to the property for memory device. Fig. 2(e) exhibits the effect of the data retention characteristics of Co-Al LDHs+Atrazine1 device. 
Under a constant voltage of $-1.5 \mathrm{~V}$, no significant current fluctuations were observed for both LRS and HRS and the ON/OFF resistance ratio could hold on above $10^{4}$ at $-1.5 \mathrm{~V}$. The LRS and HRS are also stable up to $10^{4}$ read pulses of $-1.5 \mathrm{~V}$ ( $2 \mathrm{~ms}$ in period, $1 \mathrm{~ms}$ in duration width), as exhibited in Fig. 2(f), indicating the distinguished stability of Co-Al LDHs+Atrazine1 memory device.
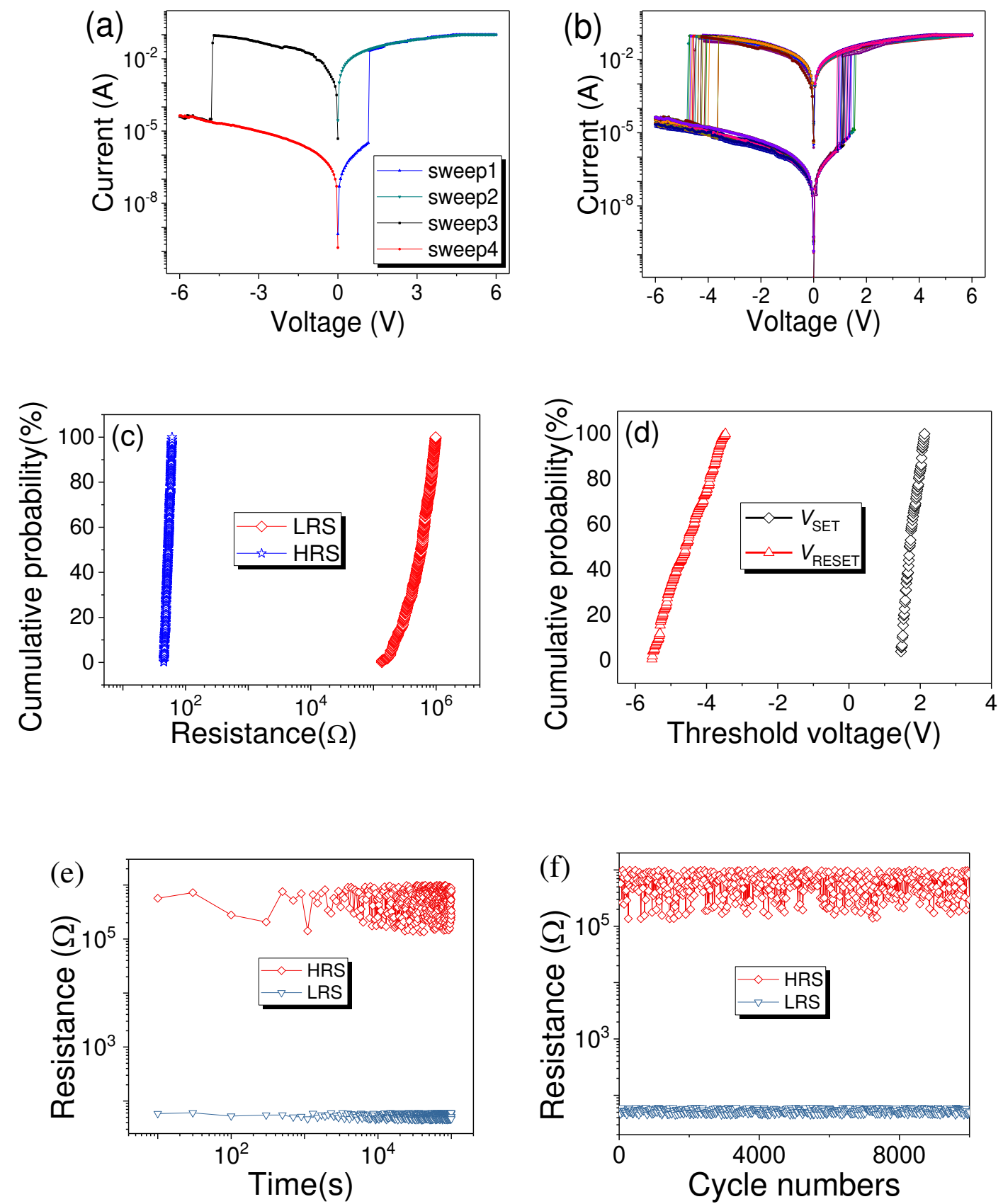

Fig. 2. (a) $I-V$ curves of Co-Al LDHs+Atrazine1 memory device. (b) Cycling endurance of resistive switching characteristics. (c) Cumulative probability for resistance in LRS and HRS. (d) Cumulative probability for threshold voltage. (e) Data retention characteristics of Co-Al LDHs+Atrazine1 device. 
(f) Endurance characteristics of Co-Al LDHs+Atrazine1 device.

As shown in Fig. 3(a), the Co-Al LDHs+Atrazine2 device shows entirely different resistive switching behaviors. The dc voltage scanning sequence applied to Co-Al LDHs+Atrazine2 device goes from $0 \mathrm{~V}$ to $+6 \mathrm{~V}$, from $0 \mathrm{~V}$ to $+6 \mathrm{~V}$, from $0 \mathrm{~V}$ to $-6 \mathrm{~V}$, and from $0 \mathrm{~V}$ to $+6 \mathrm{~V}$, the voltage sweep step is $0.01 \mathrm{~V}$. It serves to show, the Co-Al LDHs+Atrazine2 device is initially in HRS. The current holds on running at a comparatively low level during the initial stage of the first positive voltage sweep until reached to the threshold voltage of $1.37 \mathrm{~V}$. At this voltage site, the current increased abruptly from $9.57 \times 10^{-6}$ to $0.024 \mathrm{~A}$, indicating the conversion of the device from HRS to LRS. This conversion is amout to the "writing" process in a digital memory cell [37]. After this conversion, the device holds on in LRS, during the succedent positive voltage sweep2. The succedent negative voltage sweep from 0 to $-6 \mathrm{~V}$ (sweep3) does not switch the device from LRS to HRS, and the device keeps its LRS during the following forward scan (sweep4), which manifests that the HRS-to-LRS conversion is nonreversible. Once the Co-Al LDHs+Atrazine2 device is converted to LRS, it holds on there and cannot return to the original HRS, indicating its nonvolatile features. The nonreversible and nonvolatile nature of the LRS manifests that the Co-Al LDHs+Atrazine 2 device functions as the write-once read-many times (WORM) memory.

The cumulative probability for resistance in LRS and HRS were also collected for Co-Al LDHs+Atrazine2 device as exhibited in Fig. 3(c). And cumulative probability for threshold voltage for positive and negative $V_{\text {set }}$ were also analyzed for Co-Al LDHs+Atrazine2 memory device as exhibited in Fig. 3(d).

Fig. 3(e) shows the data retention properties of Co-Al LDHs+Atrazine2 device. Under a constant voltage of $0.5 \mathrm{~V}$, no significant current fluctuations were observed for LRS and HRS, and the ON/OFF resistance ratio can remain as high as about $10^{3}$ at $0.5 \mathrm{~V}$. Both of LRS and HRS are also stable up to $10^{4}$ read voltage pulses of $0.5 \mathrm{~V}$ ( $2 \mathrm{~ms}$ in period, $1 \mathrm{~ms}$ in duration width), as shown in Fig. 3(f), indicating the favourable stability of the Co-Al LDHs+Atrazine2 device. 

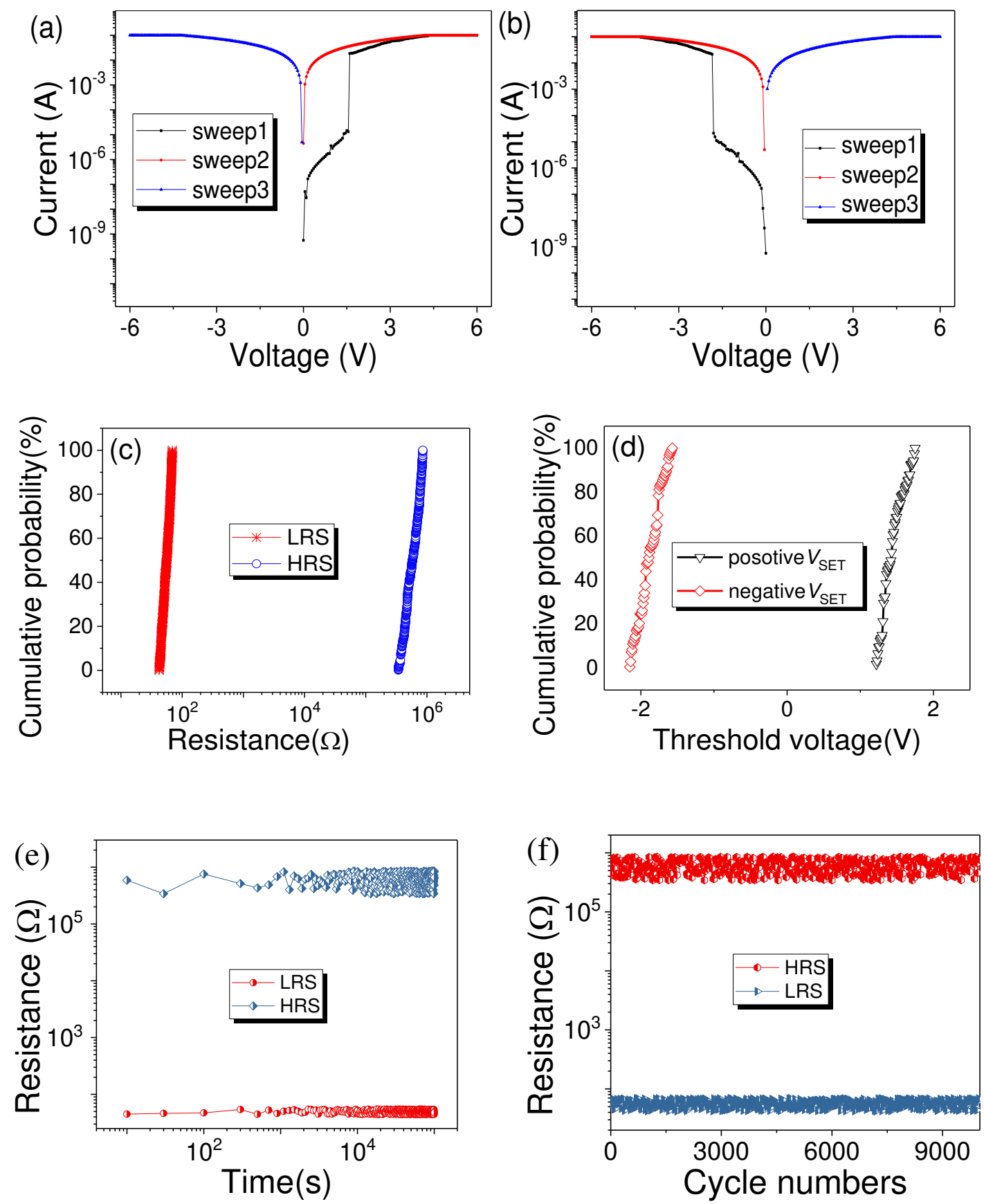

Fig. 3. (a) $I-V$ curves of Co-Al LDHs+Atrazine 2 memory device with an initial positive voltage sweep. (b) $I$ - $V$ curves of Co-Al LDHs+Atrazine2 memory device with an initial negative voltage sweep. (c) Cumulative probability for resistance in LRS and HRS. (d) Cumulative probability for switching threshold voltage. (e) Data retention characteristics of Co-Al LDHs+Atrazine2 device under a constant voltage of $0.5 \mathrm{~V}$. (f) Effect of $0.5 \mathrm{~V}$ read pulse on LRS and HRS of Co-Al LDHs+Atrazine2 device. 
For a better understanding of the resistive switching behavior, the $I$ - $V$ characteristics of devices were redrawn in log-log scale. Fig. 4 depicts its linear fitting for HRS and LRS. For Co-Al LDHs+Atrazine1 and Co-Al LDHs+Atrazine2 device, as shown in Fig. 4(a), the current-voltage curve has a linear region with a slope of 1.05 and 1.03 in LRS, which is very close to 1 . This signifies that $I-V$ relationship of the LRS defers to Ohm's law. For HRS of devices, $I-V$ curves show a linear relationship with slope of 1.07 and 1.11, and then current-voltage relationship converts to Child's law region with slope of 1.87 and 2.09 in the high voltage region, which is very convenient for space charge limited conduction (SCLC) law. This process may occur due to traps created at the interface between the electrode and the active layer. Here $\mathrm{Al}$ atoms diffused into the active layer when deposition of the top $\mathrm{Al}$ electrode resulting in the formation of impurity band for current conduction. As a result, potential band bending takes place at the electrode-active layer heterojunction by reason of the trapped electrons. A conclusion can be drawn from the above analysis, the conductive behaviors of LRS and HRS are totally different. The LRS follows Ohm's law, while the HRS complies with SCLC law.
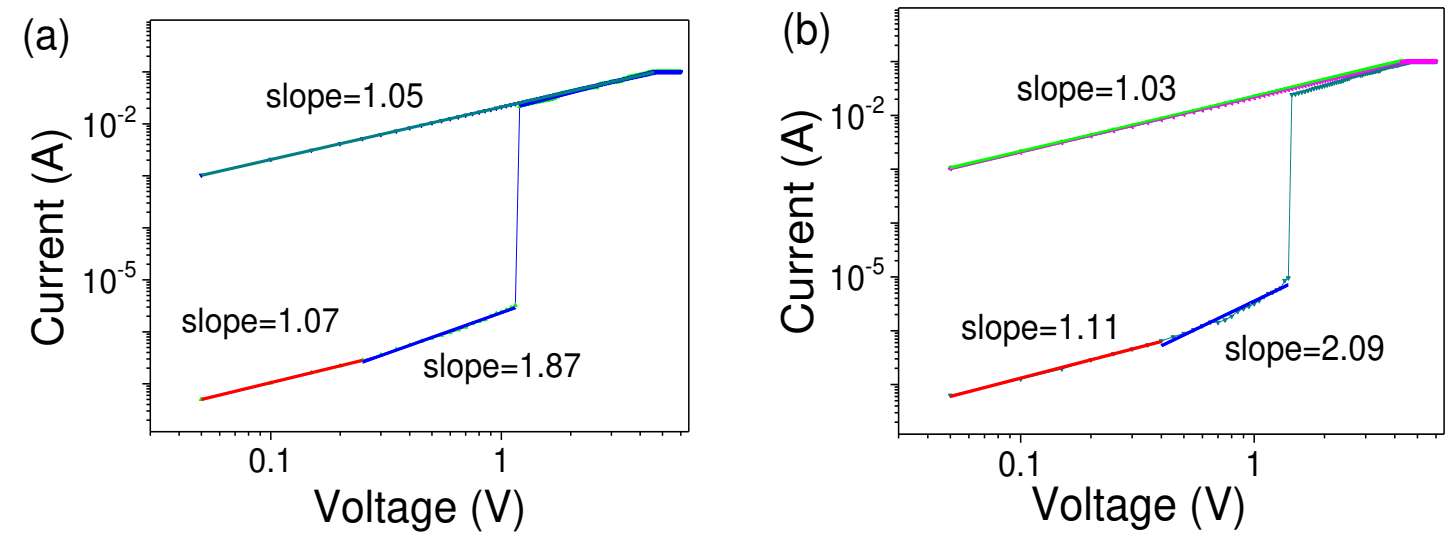

Fig. 4. Linear fitting the $I-V$ relationship of Co-Al LDHs adsorbed atrazine-based memory devices. (a) Co-Al LDHs+Atrazine1 device. (b) Co-Al LDHs+Atrazine2 device 
To study the influence of the electrical activity of top electrode, the $I-V$ curves of device with an $\mathrm{Cu}$ act as the top electrode was tested. $\mathrm{Cu}$ has more stable electrical activity than $\mathrm{Al}$. But the resistive switching behaviors were not observed in device with $\mathrm{Cu}$ top electrode as depicted in Fig. 5(a). Hence, the resistive switching of Co-Al LDHs adsorbed atrazine-based memory devices should be derived from Al filament conducting. The resistance of memory cells has almost nothing to do with area, indicating the resistive switching is a local behavior [38]. The filament conducting can be further confirmed by dependence of the resistance on the area. As exhibited in Fig. 5(b), there were no significant difference in resistance of LRS and HRS among cells with various areas. As a result, The above results manifested that resistive switching is a filament conducting in Co-Al LDHs adsorbed atrazine device.
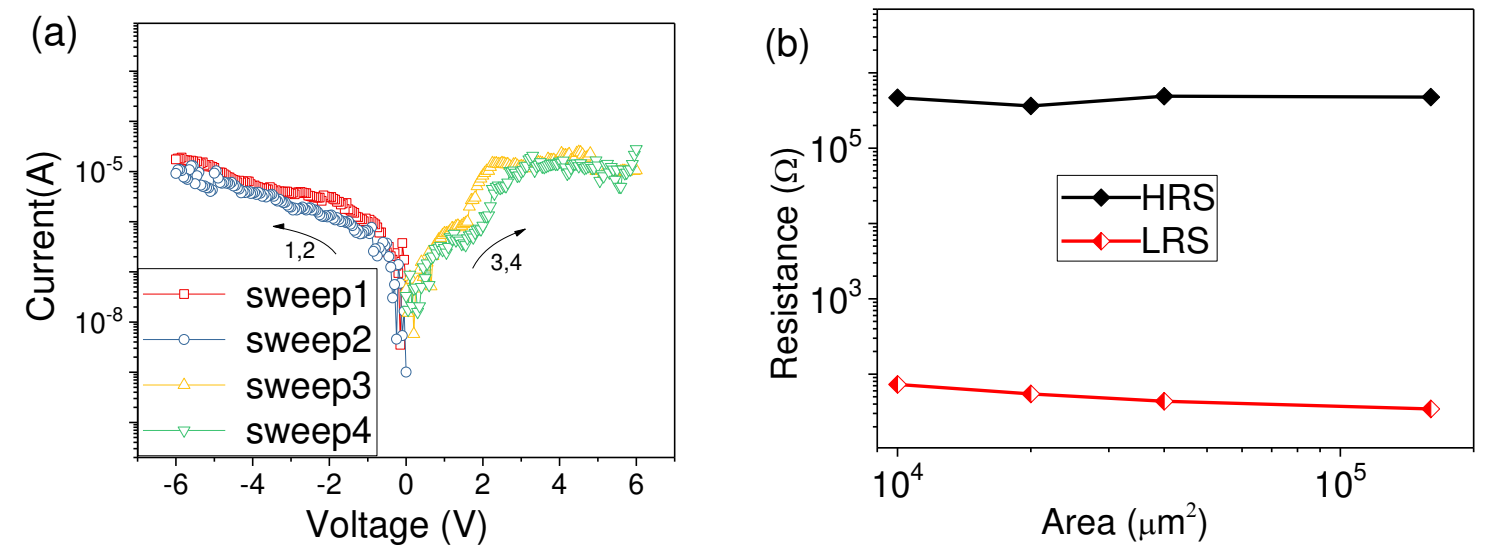

Fig. 5. (a) $I$ - $V$ curves of device with $\mathrm{Cu}$ top electrode. (b) Cell area dependence on LRS and HRS.

In order to analyze the ingredient of the conducting channel, the filament resistivity was calculated. In a general way, the diameter of the filament is $8 \sim 10 \mathrm{~nm}$ for RRAM [39]. The resistivity of the filament in our Co-Al LDHs adsorbed atrazine devices was about $2.75 \times 10^{-8} \Omega \cdot \mathrm{cm}$, because the resistance was $3.3577 \times 10^{5} \Omega\left(0.477 \times 10^{-7} \mathrm{~A}\right.$ at threshold voltage of $\left.1.22 \mathrm{~V}\right)$ and the supposed diameter and length of the conducting filament is $10 \mathrm{~nm}$ and $152 \mathrm{~nm}$ (Refer to SEM image in Fig. 
1d), respectively. The resistivity of the filament in our device is close to the resistivity of bulk Al $\left(2.83 \times 10^{-8} \Omega \cdot \mathrm{m}\right.$ at $\left.273 \mathrm{~K}\right)$. The result of the estimate shows that the conducting filament is formed by Al. To further confirm this speculation, we have tested the temperature dependence of the resistance in LRS of the device as shown in Fig. 6. According to the relationship between resistance and temperature, the resistance temperature coefficient is calculated as $4.31 \times 10^{-3} \mathrm{~K}^{-1}$ [40], as shown Fig. 6, this confirms the existence of the Al conducting filament.

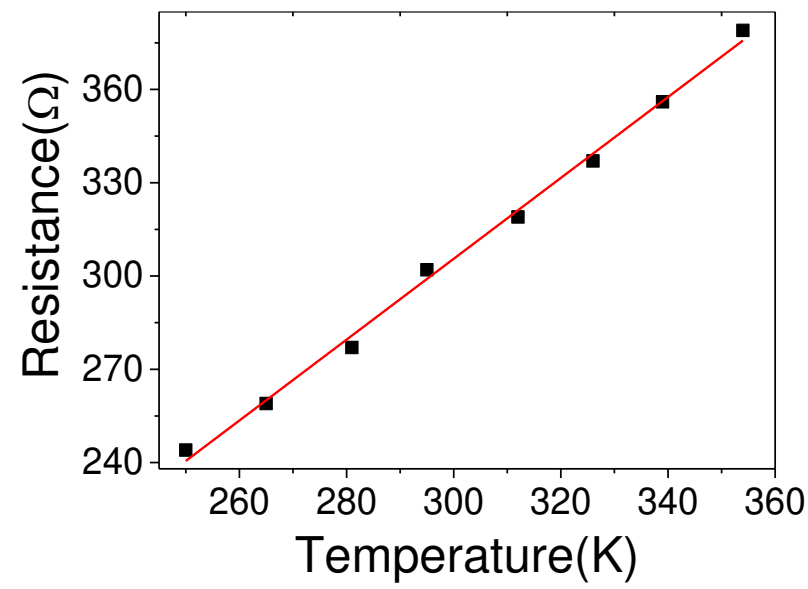

Fig. 6. Temperature dependence of the resistance in LRS of the device.

As Co-Al LDHs has a hierarchical structure, the high-speed electron transfer channel formed by the unique layered structure in its laminates can provide a channel for charge transport in the process of resistive switching and guarantee its resistive memory performance. Because Co-Al LDHs is rich in electroactive sites and large specific surface area between laminates, small-molecule atrazine is easily adsorbed on the laminates surface. In addition, the chlorine ions in atrazine has strong reducibility, these characteristics will ensure facilitated charge transfers.

Due to strong electronwithdrawing ability of Co-Al LDHs main laminate, plentiful electrons are captured by the laminate of Co-Al LDHs. Atrazine adsorbed by the host lamina could offers plentiful electron pathways throughout the adjacent plates. The Al ions introduced during thermal evaporation are easily attracted and reduced to form conductive channels between the laminate of Co-Al LDHs. Once the threshold voltage is reached, a larger number of electrons are transferred via the increased 
number of carrier pathways, leading to a distinct current increase, charge carrier transport along the electron pathways via Co-Al LDHs main laminate becomes easier, resulting in the conversion from HRS to LRS. When the opposite voltage is applied, the captured electrons are released, which resulting in disconnection of channel, hence the device is converted from LRS to HRS. Hence, Co-Al LDHs+Atrazine1 devices shows rewritable flash characteristics. With the further increase of adsorbed atrazine and the simultaneous increase in chloride ion, more charge carriers being trapped before switching, and more $\mathrm{Al}$ ions were attracted and reduced to form conductive channels, a large number of oxidizing chlorine ions are adsorbed on the Co-Al LDHs main laminate, even if the reverse voltage is applied, the carrier channel still exists, Hence, Co-Al LDHs+Atrazine2 devices shows nonvolatile WORM characteristics.

\section{Conclusion}

The Influence of adsorption small molecules atrazine on nonvolatile resistive switching behavior in Co-Al LDHs Film has been investigated. The nonvolatile resistive switching behavior of device could be adjusted in a controlled way by varying the atrazine adsorbed content in Co-Al LDHs thin films. Distinctly different nonvolatile resistive switching behaviors, WORM memory effect and rewritable memory effect are discernible from the current-voltage characteristics. Both the WORM and rewritable devices are stable under constant voltage and continuous pulse voltage stress. The nonvolatile resistive switching effects of Co-Al LDHs adsorbed atrazine have been analyzed.

\section{Declarations}

\section{Availability of data and material}

All data generated or analysed during this study are included in this published article.

\section{Competing interests}

The authors declare that they have no competing interests.

\section{Authors' contributions}

Yanmei Sun fabricated the RRAM devices; carried out the electrical measurements; drafted the 
manuscript under the instruction of Li Li. Li Li and Keying Shi supervised the work and finalized the manuscript. All authors had read and approved the final manuscript.

\section{Acknowledgements}

This work was supported by the National Natural Science Foundation of China (No. 21671060; 2167010747); the Program for Innovative Research Team in Chinese Universities (IRT1237); International Cooperation in Science and Technology Projects of China (2014DFR40480); Applied Technology Research and Development Program Foreign Cooperation Project of Heilongjiang Province (WB15C101).

\section{References}

1 Ojha S K, Kumar A, Singh A, Ojha A K (2020) Improved environmental stability of cobalt incorporated methylammonium lead iodide perovskite for resistive switching applications. Chem Phys 538: 110900.

2 Sun B, Zhou G, Guo T, Norman Zhou Y, Wu Y A (2020) Biomemristors as the next generation bioelectronics. Nano Energy 75: 104938.

3 Li D, Chen M, Sun Z, Yu P, Liu Z, Ajayan P M, Zhang Z (2017) Two-dimensional non-volatile programmable p-n junctions. Nat Nanotechnol 12: 901.

4 Tsai T -M, Lin C-C, Chen W-C, Wu C -H, Yang C -C, Tan Y -F, Wu P -Y, Huang H -C, Zhang Y -C, Sun L -C, Chou S -Y (2020) Utilizing compliance current level for controllability of resistive switching in nickel oxide thin films for resistive random-access memory. $\mathrm{J}$ Alloy Compound 826: 154126.

5 Liu C, Yan X, Song X, Ding S, Zhang D W, Zhou P (2018) A semi-floating gate memory based on vander Waals heterostructures for quasi-non-volatile applications. Nat Nanotechnol 13: 404-410. 
6 Ma G, Tang X, Zhang H, Zhong Z, Li X, Li J, Su H (2017) Ultra-high ON/OFF ratio and multi-storage on NiO resistive switching device. J Mater Sci 52: 238-246.

7 Kwon D -H, Lee S, Kang C S, Choi Y S, Kang S J, Cho H L, Sohn W, Jo J, Lee S-Y, Oh K H, Noh T W, Souza R A D, Martin M, Kim M (2019) Unraveling the Origin and Mechanism of Nanofilament Formation in Polycrystalline $\mathrm{SrTiO}_{3}$ Resistive Switching Memories. Adv Mater 31: 1901322.

8 Sun Y, Wen D (2020) Multistage resistive switching behavior organic coating films-based of memory devices. Prog Org Coat 142: 105613.

9 Sun L, Zhang Y, Hwang G, Jiang J, Kim D, Eshete Y A, Zhao R, Yang H (2018) Synaptic computation enabled by joule heating of single-layered semiconductors for sound localization. Nano Lett $18: 3229-3234$.

10 Chiappone A, Gillono M, Castellino M, Bejtka K, Rajan K, Roppolo I, Perrone D, Bocchini S, Ricciardi C, Pirri CF, Chiolerio A (2018) In situ generation of silver nanoparticles in PVDF for the development of resistive switching devices. Appl Surf Sci 455 : 418-424.

11 Yoon J, Mohammadniaei M, Choi H K, Shin M, Bharate B G, Lee T, Choi J-W (2019) Resistive switching biodevice composed of MoS2-DNA heterolayer on the gold electrode. Appl Surf Sci $478: 134-141$.

12 Lv F, Ling K, Zhong T, Liu F, Liang X, Zhu C, Liu J, Kong W (2020) Multilevel Resistive Switching Memory Based on a $\mathrm{CH}_{3} \mathrm{NH}_{3} \mathrm{PbI}{ }_{3-x} \mathrm{Cl}_{x}$ Film with Potassium Chloride Additives, Nanoscale Res Lett 15:126.

13 Sokolov A S, Jeon Y -R, Ku B, Choi C (2020) Ar ion plasma surface modification on the heterostructured $\mathrm{TaO}_{\mathrm{x}} / \mathrm{InGaZnO}$ thin films for flexible memristor synapse. J Alloy Compound $822: 153625$. 
14 Zhou G, Ren Z, Sun B, Wu J, Zou Z, Zheng S, Wang L, Duan S, Song Q (2020) Capacitive effect: An original of the resistive switching memory. Nano Energy 68: 104386.

15 Sun B, Chen Y, Xiao M, Zhou G, Ranjan S, Hou W, Zhu X, Zhao Y, Redfern S AT, Zhou Y N (2019) A Unified Capacitive-Coupled Memristive Model for the Nonpinched Current-Voltage Hysteresis Loop. Nano Lett 19: 6461-6465.

16 Li D, Chen M, Sun Z, Yu P, Liu Z, Ajayan P M, Zhang Z (2017) Two-dimensional non-volatile programmable p-n junctions. Nat Nanotechnol 12: 901.

17 Ranjan A, Raghavan N, O’Shea S J, Mei S, Bosman M, Shubhakar K, Pey K L (2018) Conductive atomic force microscope study of bipolar and threshold resistive switching in 2D hexagonal boron nitride films. Sci Rep $8: 2854$.

18 Gong Y, Lin J, Wang X, Shi G, Lei S, Lin Z, Zou X, Ye G, Vajtai R, Yakobson B I, Terrones H, Terrones M, Tay B K, Lou J, Pantelides S T, Liu Z, Zhou W, Ajayan P M (2014) Vertical and in-plane heterostructures from $\mathrm{WS}_{2} / \mathrm{MoS}_{2}$ monolayers. Nat Mater $13: 1135$.

19 Lee C -H, Lee G-H, van der Zande A M, Chen W, Li Y, Han M, Cui X, Arefe G, Nuckolls C, Heinz T F, Guo J, Hone J, Kim P (2014) Atomically thin p-n junctions with van der Waals heterointerfaces. Nat Nanotechnol 9: 676.

20 Chhowalla M, Jena D, Zhang H (2016) Two-dimensional semiconductors for transistors. Nat Rev Mater 1: 16052.

21 Sun H, Mei L, Liang J, Zhao Z (2017)Three-dimensional holey-graphene/niobia composite architectures for ultrahigh-rate energy storage. Science 356 :599-604.

22 Liu Y, Guo J, Zhu E, Liao L, Lee S-J, Ding M, Shakir I, Gambin V, Huang Y, Duan X (2018) Approaching the Schottky-Mott limit in van der Waals metal-semiconductor junctions. Nature 557: 696-700. 
23 Lin Z, Lin Z, Liu Y, Halim U, Ding M, Liu Y, Wang Y, Jia C, Chen P, Duan X, Wang C, Song F, Li M, Wan C, Huang Y, Duan X (2018) Solution-processable 2D semiconductors for highperformance large-area electronics. Nature $562: 254-258$.

24 Sun L, Zhang Y, Hwang G, Jiang J, Kim D, Eshete Y A, Zhao R, Yang H (2018) Synaptic computation enabled by joule heating of single-layered semiconductors for sound localization. Nano Lett 18: 3229-3234.

25 Ge R, Wu X, Kim M, Shi J, Sonde S, Tao L, Zhang Y, Lee J C, Akinwande D (2018) Atomristor: nonvolatile resistance switching in atomic sheets of transition metal dichalcogenides. Nano Lett $18: 434-441$.

26 Sun L, Yu H, Wang D, Jiang J, Kim D, Kim H, Zheng S, Zhao M, Ge Q, Yang H (2018) Selective growth of monolayer semiconductors for diverse synaptic junctions. 2D Mater 6 : 015029.

27 Qiu H, Pan L, Yao Z, Li J, Shi Y, Wang X (2012) Electrical characterization of back-gated bi-layer $\mathrm{MoS}_{2}$ fieldeffect transistors and the effect of ambient on their performances. Appl Phys Lett 100: 123104.

28 Shao M, Han J, Wei M, Evans D G, Duan X (2011) The synthesis of hierarchical Zn-Ti layered double hydroxide for efficient visible-light photocatalysis. Chem Eng J 168 : 519-524.

29 Zhou G, Ren Z, Wang L, Wu J, Sun B, Zhou A, Zhang G, Zheng S, Duan S, Song Q (2019) Resistive switching memory integrated with amorphous carbon-based nanogenerators for self-powered device. Nano Energy 63 : 103793.

30 Chiu C H, Huang C W, Hsieh Y H, Chen J Y, Chang C F (2017) In-situ TEM observation of Multilevel Storage Behavior in low power FeRAM device. Nano Energy 34 : 103-110. 
31 Sun L, Zhang Y, Hwang G, Jiang J, Kim D, Eshete YA, Yang H (2018) Synaptic Computation Enabled by Joule Heating of Single-Layered Semiconductors for Sound Localization. Nano Lett $18: 3229-3234$.

32 Sun L, Zhang Y, Han G, Hwang G, Jiang J, Joo B, Watanabe K, Taniguchi T, Kim Y-M, Yu W J, Kong B-S, Zhao R, Yan H (2019) Self-selective van der Waals heterostructures for large scale memory array. Nat Commun 10: 3161.

33 Liu G, Ling Q-D, Yeow E, Teo H, Zhu C-X, Chan D S-H, Neoh K-G, Kang E-T (2009) Electrical Conductance Tuning and Bistable Switching in Poly:(N-vinylcarbazole) Carbon Nanotube Composite Films. ACS Nano 3: 1929-1937.

34 Liu Z, Teng L, Ma L, Liu Y, Zhang X, Xue J, Ikram M, Ullah M, Li L, Shi K (2019) Porous 3D flower-like CoAl-LDH nanocomposite with excellent performance for $\mathrm{NO}_{2}$ detection at room temperature. RSC Adv 9: 21911-21921.

35 Sun H, Chu Z, Hong D, Zhang G, Xie Y, Li L, Shi K (2016) Three-dimensional hierarchical flower-like Mg-Al-layered double hydroxides: Fabrication, characterization and enhanced sensing properties to NOx at room temperature. J Alloy Compound 658: 561-568.

36 Shi L, Ye H, Liu W, Tian G, Qi S, Wu D (2013)Tuning the electrical memory characteristics from WORM to flash by a- and b-substitution of the electron-donating naphthylamine moieties in functional polyimides. J Mater Chem C 1: 7387-7399.

37 Shulaker, M M et al (2017) Three-dimensional integration of nanotechnologies for computing and data storage on a single chip. Nature 547: 74.

38 Sawa A (2008) Resistive switching in transition metal oxides. Mater Today 11 : 28-36.

39 Wei Z, Takagi T, Kanzawa Y, Katoh Y, Ninomiya T, Kawai K, Muraoka S, Mitani S, Katayama K, Fujii S (2011) Demonstration of High-Density ReRAM Ensuring 10-Year Retention at $85{ }^{\circ} \mathrm{C}$ Based on a Newly Developed Reliability Model. Electron Devices Meeting :(IEDM) : 
3141-3144.

40 Sun Y, Wen D, Sun F (2019) Eliminating Negative-set Behavior by Adding A Graphene Blocking Layer in Resistive Switching Memory Devices Based on Epoxy Resin. Appl Phys Express 12: 074006. 


\section{Figures}
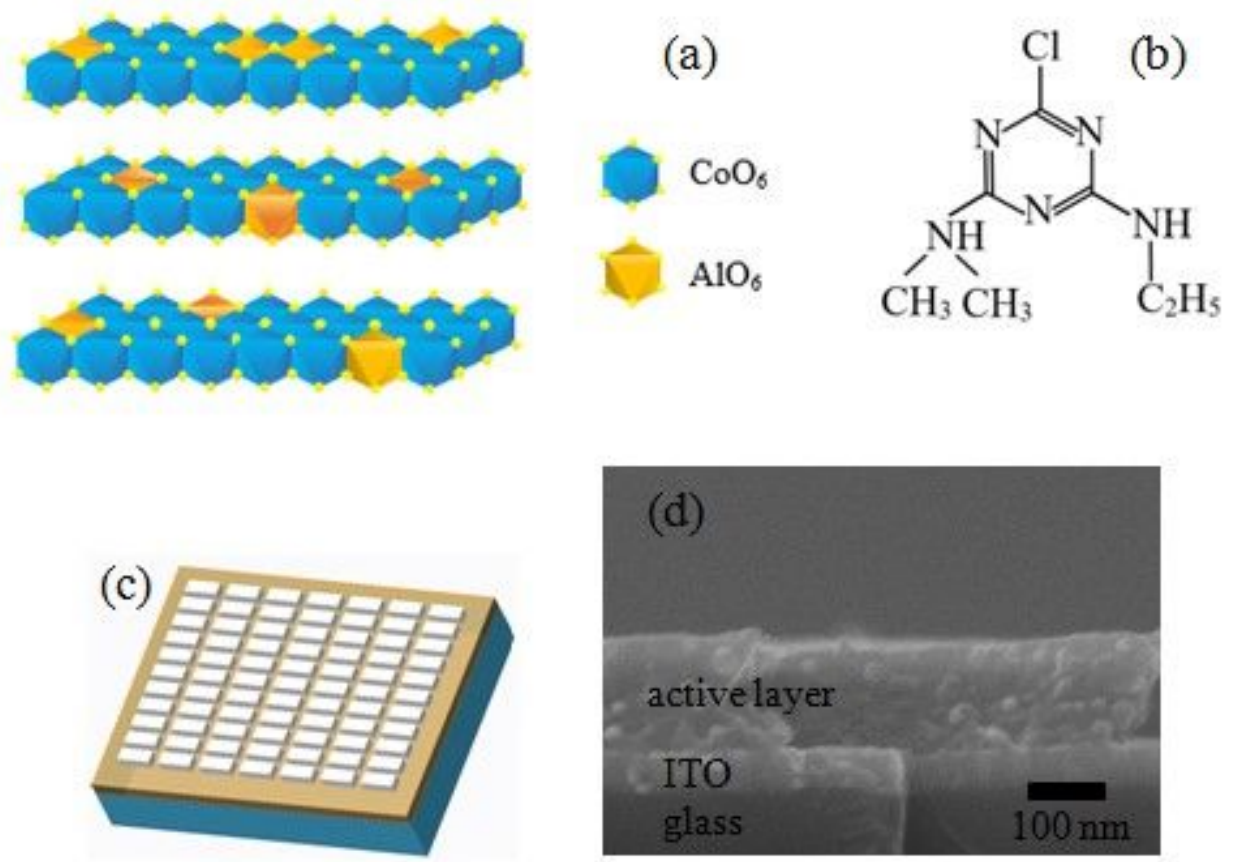

\section{Figure 1}

(a) Chemical structure diagram of Co-AI LDHs. (b) Chemical structure of atrazine. (c) Schematic structure of the Co-Al LDHs adsorbed atrazine-based memory device. (d) The cross section of the resistive switching layer. 

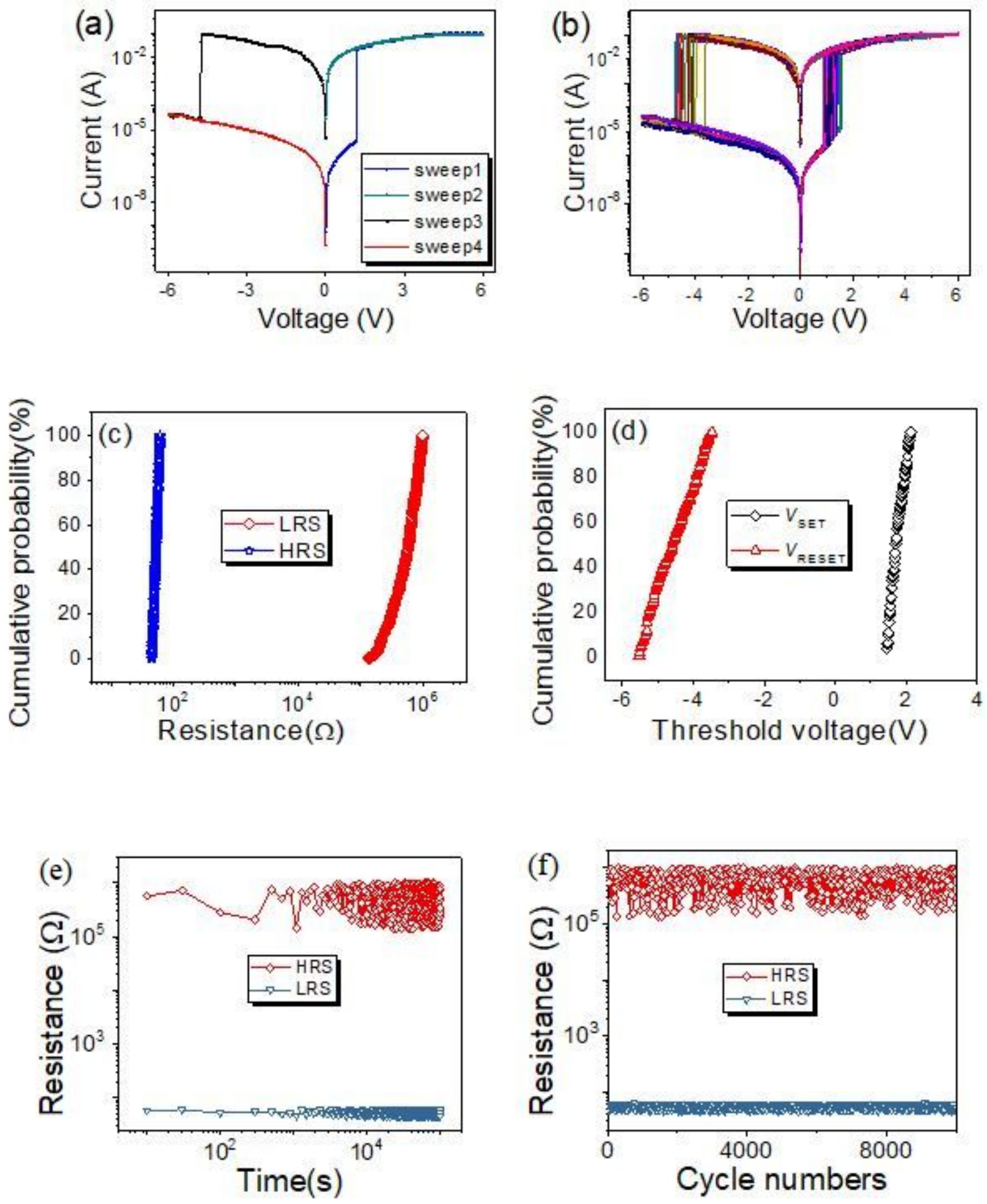

Figure 2

(a) I-V curves of Co-Al LDHs+Atrazine1 memory device. (b) Cycling endurance of resistive switching characteristics. (c) Cumulative probability for resistance in LRS and HRS. (d) Cumulative probability for threshold voltage. (e) Data retention characteristics of Co-Al LDHs+Atrazine1 device. (f) Endurance characteristics of Co-Al LDHs+Atrazine1 device. 

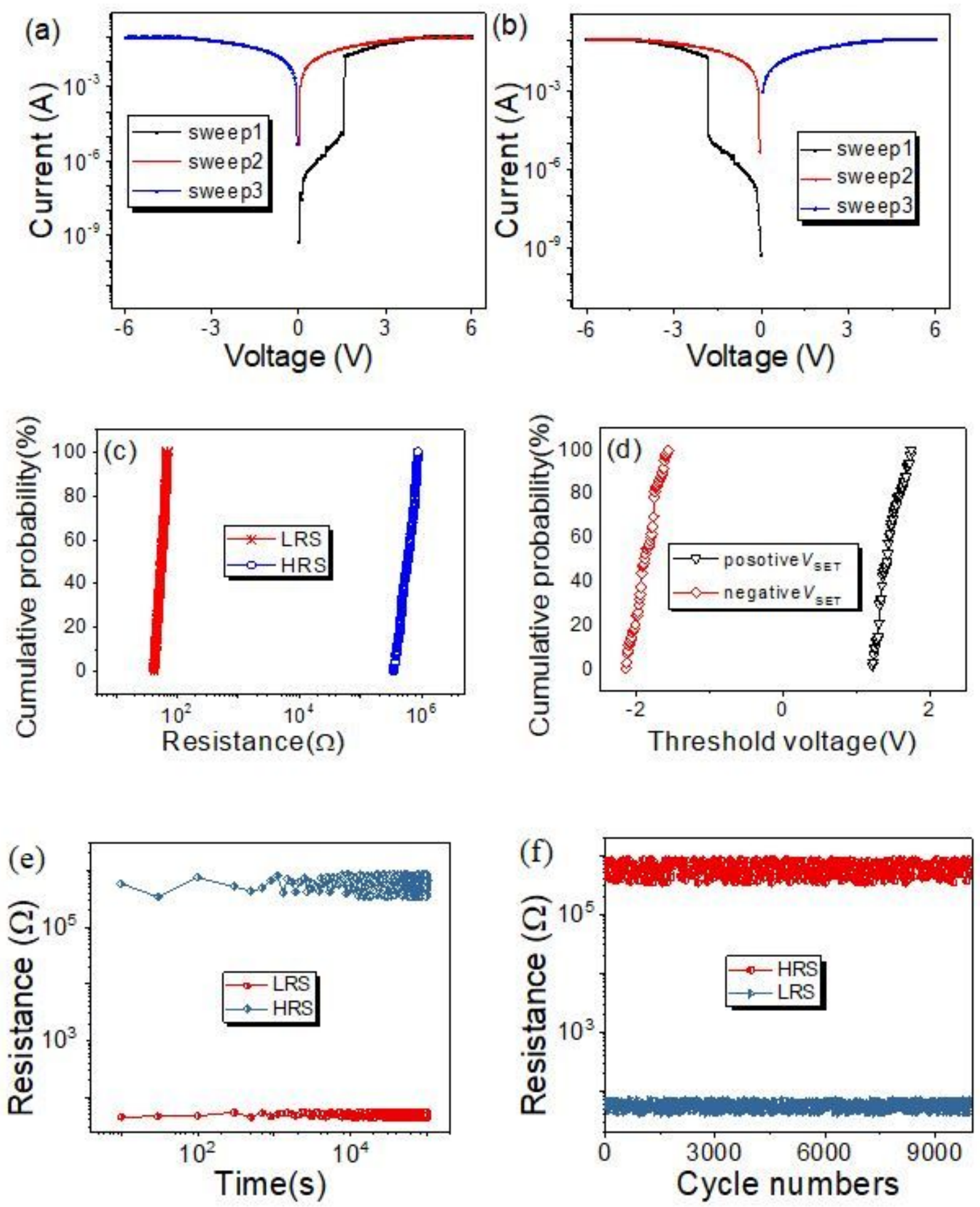

Figure 3

(a) I-V curves of Co-Al LDHs+Atrazine2 memory device with an initial positive voltage sweep. (b) I-V curves of Co-Al LDHs+Atrazine2 memory device with an initial negative voltage sweep. (c) Cumulative probability for resistance in LRS and HRS. (d) Cumulative probability for switching threshold voltage. (e) Data retention characteristics of Co-Al LDHs+Atrazine2 device under a constant voltage of $0.5 \mathrm{~V}$. (f) Effect of $0.5 \mathrm{~V}$ read pulse on LRS and HRS of Co-Al LDHs+Atrazine2 device. 

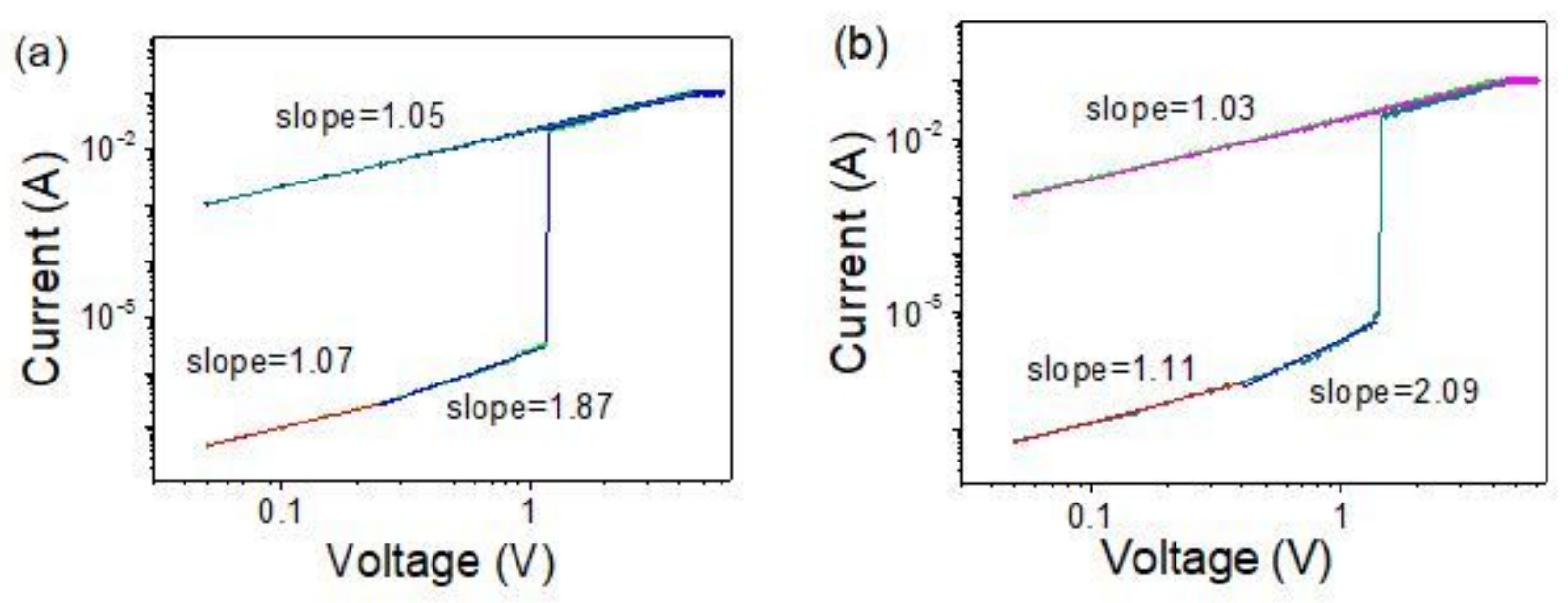

\section{Figure 4}

Linear fitting the I-V relationship of Co-AI LDHs adsorbed atrazine-based memory devices. (a) Co-Al LDHs+Atrazine1 device. (b) Co-Al LDHs+Atrazine2 device
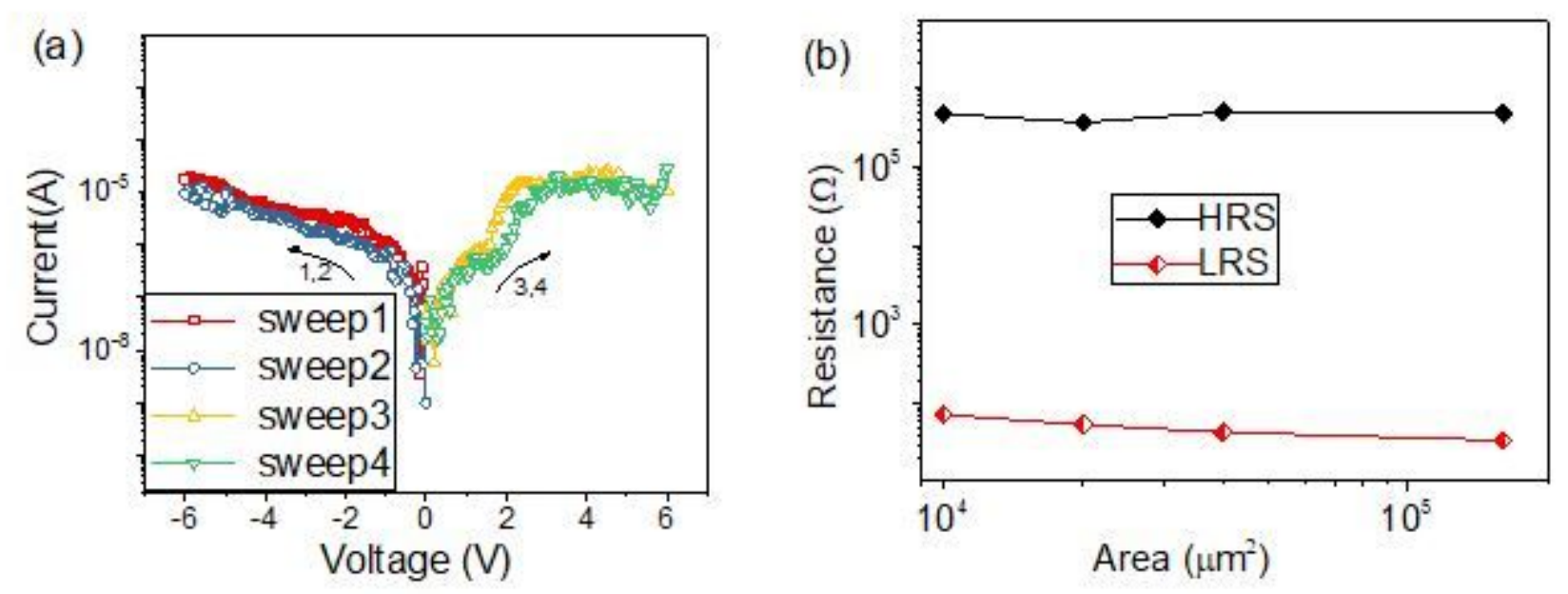

Figure 5

(a) I-V curves of device with Cu top electrode. (b) Cell area dependence on LRS and HRS. 


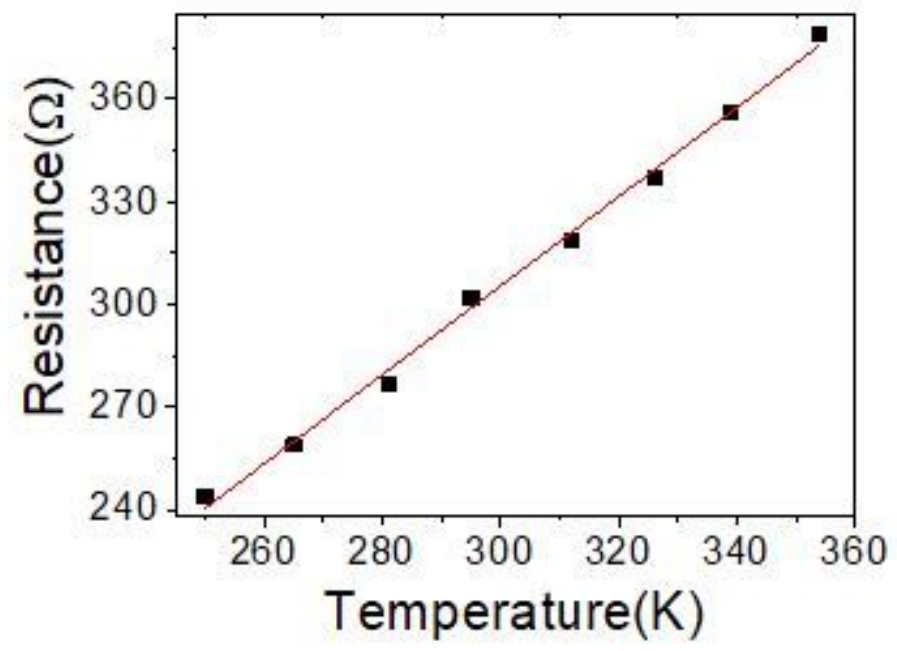

Figure 6

Temperature dependence of the resistance in LRS of the device. 